\title{
Multi-waveband photons and high energy neutrinos from the Crab nebula
}

\author{
X. C. Yang and L. Zhang
}

\author{
Department of Physics, Yunnan University, 650091 Kunming, PR China \\ e-mail: lizhang@ynu.edu.cn
}

Received 28 August 2008 / Accepted 12 January 2009

\begin{abstract}
We study multi-waveband photon emission properties and possible high-energy neutrino emission from the Crab nebula produced by pulsar's accelerated heavy nuclei. Heavy nuclei are accelerated in the outer magnetosphere of the Crab pulsar, and then suffer partial photo-disintegration in the non-thermal radiation fields of the pulsar's outer magnetosphere. The products (protons and neutrons) of the photo-disintegration and surviving heavier nuclei are injected into the expanding supernova remnant in which the surviving heavier nuclei are further accelerated in the pulsar wind zone. In such a case, relativistic hadrons and leptons are produced; the hadrons consist of direct protons and the protons produced through neutron decay in nuclei disintegration process, the leptons consist of positrons accelerated in a realistic magneto-sonic lepton-heavy iron plasma shock, electrons from the neutron decay, direct electrons, and secondary electron/positron pairs in a proton-proton interaction. We calculate the photo-disintegration rate for a nucleus of atomic number A releasing $i$ nucleons and the hadronic and leptonic spectra in the Crab nebula. We then calculate the multi-waveband nonthermal photon spectrum and possible high-energy neutrino spectrum of the Crab nebula. Our results indicate that the photon spectrum up to $\sim 10 \mathrm{TeV}$ has a leptonic origin, but it is difficult to distinguish leptonic and hadronic origins of the spectrum with energy greater than $10 \mathrm{TeV}$.
\end{abstract}

Key words. radiation mechanisms: non-thermal - ISM: individual objects: Crab nebula - gamma rays: theory

\section{Introduction}

Pulsar wind nebulae (PWNe) are generally believed to be a kind of Galactic cosmic ray source. The Crab nebula, being a well established gamma-ray source with a complex spectrum extending up to at least $\sim 80 \mathrm{TeV}$ energies, is a possible object in which the particles can be accelerated up to $\sim 10^{15-16} \mathrm{eV}$ (e.g. de Jager \& Harding 1992; Aharonian \& Atoyan 1995). For the Crab nebula, however, there are two possible kinds of models to explain the observed $\mathrm{TeV}$ emission properties: leptonic origin models (e.g. Jager \& Harding 1992; Atoyan \& Aharonian 1996; Zhang et al. 2008) and hadronic origin models (e.g. Cheng et al. 1990; Atoyan \& Aharonian 1996; Bednarek \& Prothero 1997; Bednarek \& Bartosik 2003; a review see Bednarek 2007).

Photon emission with energy up to several tens of $\mathrm{TeV}$ from the Crab nebula has been usually interpreted with the framework of synchrotron self-Compton (SSC) model. In such a model, relativistic leptons inside the nebula produce soft synchrotron photons, and these soft photons are next up-scattered by the same leptons to gamma-ray energies through an inverse Compton (IC) process, possible soft photons also include microwave background radiation (MBR), infrared or the optical background inside the nebula. This model has been successfully applied to the best studied TeV gamma-ray source-the Crab nebula and other TeV PWNe (de Jager \& Harding 1992; Atoyan \& Aharonian 1996; Zhang et al. 2008). On the other hand, the selfconsistent, time dependent hadronic-leptonic model has been proposed to account for the high energy processes inside PWNe (e.g. Bednarek \& Prothero 1997; Bednarek \& Bartosik 2003, 2005). In such a model, heavy nuclei are accelerated in the pulsar magnetosphere, and then suffer partial photo-disintegration in the non-thermal radiation fields of pulsar's outer magnetosphere, leading to the production of relativistic protons and neutrons. These protons and neutrons, as well as surviving heavier nuclei, are injected into the expanding supernova remnant. Inside the remnant, relativistic hadrons consist of direct protons and the protons produced through neutron decay in the nuclei disintegration process, the leptons are excited by the relativistic nuclei and consist of electrons from the neutron decay, positrons accelerated in the realistic magneto-sonic lepton-heavy iron plasma shock, and secondary electron/positron pairs in the protonproton interaction.

In this paper we study the production of non-thermal photons and high-energy neutrinos in the Crab nebula based on the model of Bednarek \& Protheroe (1997) and Bednarek \& Bartosik (2003). In our treatment, we consider the relativistic proton spectrum from both direct proton production and indirect proton production from neutron decay through the disintegration process. We also consider four kinds of leptons: the positrons accelerated in a realistic magneto-sonic lepton-heavy iron plasma shock (Hoshino \& Arons 1991; Hoshino et al. 1992; Gallant \& Arons 1994), electrons accompanying neutron decay, direct electrons, and secondary $\mathrm{e}^{ \pm}$pairs in proton-proton $(p p)$ collisions (We call them nuclei-excited leptons except for the direct electrons, which accompany the nuclei). Different from the work in Bednarek \& Bartosik (2003), we include the direct proton contribution which dominates the high-energy end of the proton spectrum, and use the complete form of the positron spectrum (i.e. a Maxwellian distribution plus a power-law tail) given by Hishino et al. (1992). Using spectra of both hadrons and leptons, we calculate the non-thermal spectrum of the Crab nebula, where the inclusive cross sections of stable secondary particles produced by proton-proton interaction, in parameterized formulae given 
by Kelner et al. (2006), are used. The organization of this paper is as follows. We describe the model in Sect. 2, and apply the model to the Crab nebula in Sect. 3. We give our conclusions and discussion in Sect. 4.

\section{Leptons and hadrons produced in the nebula}

We describe the basic physics processes which produce nonthermal emission from the Crab nebula in the framework of the hadronic-leptonic model.

\subsection{Nucleon injection in the pulsar magnetosphere}

Rapidly rotating neutron stars are known to convert a significant part of their rotation energy into radiation and the emission is observable as a periodic signal. It is possible that rotating magnetospheres of a neuron star can accelerate not only leptons but also nuclei extracted from the positively charged polar cap region (Bednarek \& Bartosik 2004). The presence of heavy nuclei can explain the high energy phenomena observed around a pulsar (Gil et al. 2003). The amount of irons accelerated in the inner magnetosphere and pulsar wind zone, and injected in the pulsar wind nebula, can be written as (Bednarek \& Protheroe 1997)

$\dot{N}_{\mathrm{Fe}}=\xi L / Z \Phi(B, P)$

where $\xi$ is the fraction of total dissipated power taken by relativistic nuclei accelerated in the outer gap, $Z=26$ is the charge number of iron, $B$ is the surface magnetic field, $P$ is the pulsar period, $L$ is the rate of kinetic rotational energy dissipated

$L=4 \pi^{2} I \frac{\dot{p}}{p^{3}}$,

and

$\Phi(B, P) \approx 5 \times 10^{16}\left(\frac{B}{4 \times 10^{12} \mathrm{G}}\right)\left(\frac{P}{\mathrm{~s}}\right)^{4 / 3} V$

is the potential difference across the outer gap (Cheng et al. 1990), where $I$ is the neutron star moment of inertia which is assumed to be $1 \times 10^{45} \mathrm{~g} \mathrm{~cm}^{2}, B$ is the surface magnetic field strength which is inferred from the pulsar period $P$ and period derivative $\dot{P}$. The spin period evolves from $P_{0}$ as

$P=P_{0}\left(1+\frac{t}{\tau_{0}}\right)^{\frac{1}{n-1}}$,

where $n$ is the braking index (e.g. Gaensler \& Slane 2006).

\subsection{Photodisintegration of nuclei in the pulsar soft photon field}

It has been proposed that there is the possibility of photodisintegration of Fe nuclei during their propagation through the soft photon field from the Crab pulsar (Bednarek \& Protheroe 1997). The Fe nuclei escape from the polar cap surface and move along magnetic field lines to enter the outer gap where they can be accelerated in the outer gap potential, as in the model of Cheng et al. (1986a). Since nuclei have much larger Larmor radii and much lower synchrotron and curvature radiations, they easily propagate out across the field lines along the outer gap. The nuclei suffer disintegration in collisions with the nonthermal radiation field in the outer gap produced by accelerated electron and positron cascades; the dimension of the outer gap is of the order of the radius of the light cylinder, namely, $l_{\text {gap }} \approx 1.5 \times 10^{8} \mathrm{~cm}$

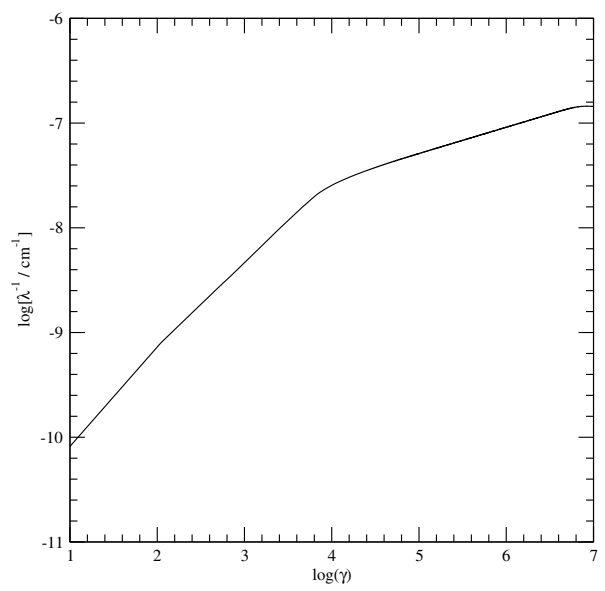

Fig. 1. Reciprocal mean free path for ${ }_{56}^{26} \mathrm{Fe}$ in the Crab magnetosphere.

(Cheng et al. 1986b). A single proton or neutron emits in each nucleus disintegration, with the same Lorentz factor of the nucleus. The nucleons injected into the nebula, both the directly emitted protons and protons from neutron decay, are trapped and accumulate in the nebula.

The photo-disintegration rate for a nucleus of atomic number $A$ releasing $i$ nucleons can be written as

$R_{\mathrm{A}, i}=\frac{1}{2 \gamma^{2}} \int_{0}^{\infty} \epsilon^{-2} n(\epsilon) \mathrm{d} \epsilon \int_{0}^{2 \gamma \epsilon} \mathrm{d} \epsilon^{\prime} \epsilon^{\prime} \sigma_{\mathrm{A}, i}\left(\epsilon^{\prime}\right)$,

where $\gamma$ is the Lorentz factor of the nucleus, and $\epsilon, \epsilon^{\prime}$ the photon energies in the observer and nucleus rest frames respectively. $n(\epsilon)$ is the soft photon field in the pulsar magnetosphere and for the Crab pulsar is given by (Cheng et al. 1986)

$n(\epsilon)=n_{0} \epsilon^{-v}$,

where $n_{0}$ is estimated using $n\left(\epsilon=m_{\mathrm{e}} c^{2}\right) \approx 3 \times 10^{15} \mathrm{~cm}^{-3}, v=2.2$ for $h v \geq 1, v=1.8$ for $3 \times 10^{-3} \leq h v \leq 1, v=1.25$ for $3 \times 10^{-6} \leq h v \leq 3 \times 10^{-3}$, and $v=0.66$ for $h v \leq 3 \times 10^{-6}$. In Eq. (4), $\sigma_{\mathrm{A}, i}\left(\epsilon^{\prime}\right)$ is the cross section of the photodisintegration; here we use the Gaussian parameterization for the cross section given by Puget et al. (1976) (also see the revision of Stecker $\&$ Salamon 1999). In such a soft photon field, we calculate a reciprocal mean free path $\lambda=c / R_{\mathrm{A}, i}$ and show the reciprocal mean free path of ${ }_{56}^{26} \mathrm{Fe}$ in Fig. 1. The result is similar to that of the Lorentian curve approximation (Karakula \& Tkaczyk 1993; Bednarek \& Protheroe 1997).

\subsection{Proton spectrum}

When the photodisintegration rate for a nucleus of atomic number $A$ releasing $i$ nucleons is given, the number of the $i$ nucleons per unit energy can be estimated. For the treatment of entangled acceleration and disintegration process, we assume the nuclear Lorentz factor at position $s$ is $\gamma_{\mathrm{A}}(s)$, where $s$ is distance relative to the inner point of outer gap. For simplicity, the parallel component of the electrical field is assumed constant, as $E_{\|} \approx \Phi(B, P) / l_{\text {gap }}$, so we have $\gamma_{\mathrm{A}}(s) \approx\left(Z E_{\|} s\right) /\left(A m_{\mathrm{p}} c^{2}\right)$ and $\mathrm{d} s / \mathrm{d} \gamma_{\mathrm{A}} \approx\left(A m_{\mathrm{p}} c^{2} l_{\text {gap }}\right) /(Z \Phi(B, P))$. Therefore, the number of $i$ nucleons at energy $E_{i}$ per unit energy per nucleus is approximated as

$N_{i}\left(\gamma_{i}(t), t\right)=\frac{\mathrm{d} N}{\mathrm{~d} \gamma_{i}}=\frac{\mathrm{d} s}{\mathrm{~d} \gamma_{\mathrm{A}}} \frac{1}{\lambda}=\frac{A l_{\mathrm{gap}} m_{\mathrm{p}} c R_{\mathrm{A}, i}}{Z \Phi(B, P)}$,

where $i=n, p$, and $c$ is the light speed. 
We now can construct and compute the total proton spectrum present in the Crab nebula including both the contribution of neutrons and protons. The present spectrum of protons from accelerated nuclei disintegrating in the nebula is the result of trapping and accumulation from the birth of pulsar to the present time and depends on the pulsar evolution since its birth, collisions and absorption by matter; this is estimated by optical depth. Using initial period $P_{0}=19 \mathrm{~ms}$ and braking index $n=2.5$ for the Crab pulsar (Lyne et al. 1993), the pulsar period evolves as Eq. (3). During the evolution of the Crab pulsar, the energy losses are mainly due to $p p$ collision and adiabatic expansion. For the present Lorentz factor $\gamma_{\mathrm{p}}$ at time $t_{\mathrm{CN}}$, its Lorentz factor at time $t$ can be estimated as (Bednarek \& Protheroe 1997)

$\gamma_{\mathrm{p}}(t) \approx \gamma_{\mathrm{p}} \frac{t+t_{\mathrm{CN}}}{2 t K^{\tau_{\mathrm{pp}}(t)}}$,

where the $\tau_{\mathrm{pp}}(t)$ is the optical depth for the collision of protons with matter between $t$ and $t_{\mathrm{CN}}$ which can be approximated by $\tau_{\mathrm{pp}}(t) \approx 1.3 \times 10^{17} M_{1} v_{8}^{-3}\left(t^{-2}-t_{\mathrm{CN}}^{-2}\right)$, and $K$ is the inelasticity coefficient in proton-proton collisions. In such a case, the energy spectra of protons extracted from $\mathrm{Fe}$ nuclei at an early time $t$ are given by $\mathrm{d} N_{\mathrm{p}}\left(\gamma_{\mathrm{p}}, t_{\mathrm{CN}}\right) / \mathrm{d} E=[\mathrm{d} E(t) / \mathrm{d} E]\left[\mathrm{d} N_{\mathrm{p}} / \mathrm{d} E(t)\right]$, where $\mathrm{d} E(t) / \mathrm{d} E=\gamma_{\mathrm{p}}(t) / \gamma_{\mathrm{p}}$ is estimated using Eq. (7). The optical depth due to absorption by matter can be expressed as $\tau_{\mathrm{nH}} \approx \sigma_{\mathrm{pp}} n_{\mathrm{H}} r \approx 8.6 \times 10^{14} M_{1} v_{8}^{-2} t^{-2}$, where $M=M_{1} M_{\odot}$ is the mass ejected during the supernova explosion in units of solar mass, $n_{\mathrm{H}}$ is the number density of matter, and $v=10^{8} v_{8} \mathrm{~cm} \mathrm{~s}^{-1}$ is the expansion velocity of the nebula; $v=2000 \mathrm{~km} \mathrm{~s}^{-1}$ is used here. Following Bednareck \& Protheroe (1997), the proton spectrum from neutron decay is

$$
\begin{aligned}
& {\left[\frac{\mathrm{d} N_{\mathrm{p}}\left(\gamma_{\mathrm{p}}, t_{\mathrm{CN}}\right)}{\mathrm{d} E}\right]_{1}=\frac{1}{\gamma_{\mathrm{p}} m_{\mathrm{p}} c^{2}} \int_{0}^{t_{\mathrm{CN}}} \mathrm{d} t \dot{N}_{\mathrm{Fe}}(t) \mathrm{e}^{-\tau_{\mathrm{nH}}(t)}} \\
& \times\left[N_{\mathrm{n}}\left(\gamma_{\mathrm{p}}(t), t\right) \gamma_{\mathrm{p}}(t)\left[1-\exp \left(v t / c \gamma_{\mathrm{p}}(t) \tau_{\mathrm{n}}\right)\right]\right. \\
& \left.\quad+\int_{t}^{t_{\mathrm{CN}}} \mathrm{d} t^{\prime} N_{\mathrm{n}}\left(\gamma_{\mathrm{p}}\left(t^{\prime}\right), t^{\prime}\right) \gamma_{\mathrm{p}}\left(t^{\prime}\right) \frac{v \exp \left(v t^{\prime} / c \gamma_{\mathrm{p}}\left(t^{\prime}\right) \tau_{\mathrm{n}}\right)}{c \gamma_{\mathrm{p}}\left(t^{\prime}\right) \tau_{\mathrm{n}}}\right] .
\end{aligned}
$$

The first term is the contribution from neutrons initially decaying inside the nebula, and the second term gives the contribution of neutron initially decaying outside the nebula.

The direct contribution of proton emission is different from the disintegrated neutron component; all successive protons emitted from nuclei disintegrating and accelerating in the outer gap could be accelerated continuously and achieve almost the same energy determined by the outer gap potential $E_{\mathrm{p}}(t)=$ $Z / A \Phi(B, P(t))$, therefore ultimately the direct proton number $\mathrm{d} N$ with energy $E_{\mathrm{p}}(t)$ at pulsar period $P$ is the sum of the differential energy spectra of disintegrated protons from $E=0$ to $E=E_{\mathrm{p}}(t)$, $\mathrm{d} N=\dot{N}_{\mathrm{Fe}} \mathrm{d} t \int_{0}^{E_{\mathrm{p}}(t)} \mathrm{d} E^{\prime} N_{\mathrm{p}}\left(E^{\prime}\right)$, i.e.

$\frac{\mathrm{d} N}{\mathrm{~d} E_{\mathrm{p}}(t)}=\dot{N}_{\mathrm{Fe}} \frac{\mathrm{d} t}{\mathrm{~d} E_{\mathrm{p}}(t)} \int_{0}^{E_{\mathrm{p}}(t)} \mathrm{d} E^{\prime} N_{\mathrm{p}}\left(E^{\prime}\right)$.

Taking account of the energy loss, we reach the direct proton contribution of the energy spectrum as

$$
\begin{aligned}
& {\left[\frac{\mathrm{d} N_{\mathrm{p}}\left(\gamma_{\mathrm{p}}, t_{\mathrm{CN}}\right)}{\mathrm{d} E}\right]_{2}=\frac{\gamma_{\mathrm{p}}(t)}{\gamma_{\mathrm{p}}} \frac{\mathrm{d} N}{\mathrm{~d} E_{\mathrm{p}}(t)} \mathrm{e}^{-\tau_{\mathrm{nH}}(t)}} \\
& =\frac{\gamma_{\mathrm{p}}(t)}{\gamma_{\mathrm{p}}} \dot{N}_{\mathrm{Fe}}(t) \frac{\mathrm{d} t}{\mathrm{~d} P} \frac{\mathrm{d} P}{\mathrm{~d} E_{\mathrm{p}}(t)} \mathrm{e}^{-\tau_{\mathrm{nH}}(t)} \int_{0}^{\gamma_{\mathrm{p}}(t)} \mathrm{d} \gamma^{\prime} N_{\mathrm{p}}\left(\gamma^{\prime}\right),
\end{aligned}
$$

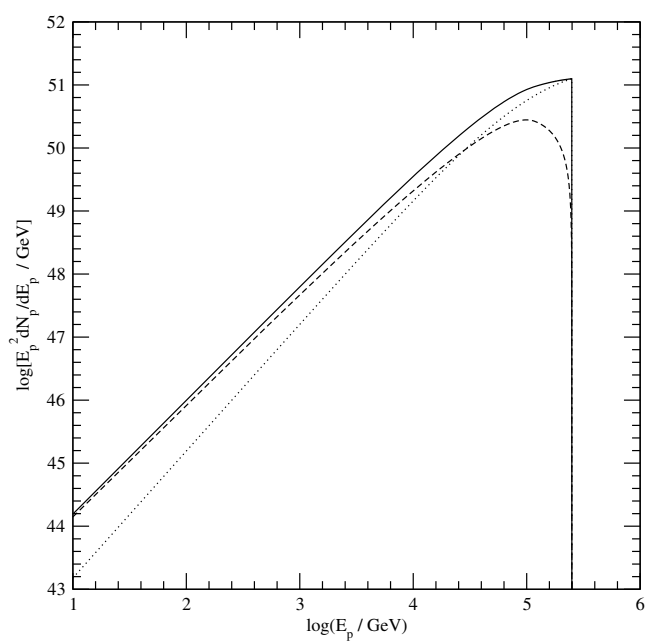

Fig. 2. Present proton spectrum in the Crab nebula. The solid line represents the total proton spectrum, dashed and dotted lines represent the spectra of the protons from neutron decay (Eq. (8)) and the direct protons (Eq. (10)).

where $N_{\mathrm{p}}\left(\gamma^{\prime}\right)$ is given by Eq. (6), $\mathrm{d} P / \mathrm{d} E_{\mathrm{p}}(t)$ and $\mathrm{d} t / \mathrm{d} P$ can be derived using Eqs. (2) and (3), which are $\mathrm{d} P / \mathrm{d} E_{\mathrm{p}}(t)=$ $(3 / 20) \times 10^{-16}(A / Z)\left(4 \times 10^{12} \mathrm{G} / B\right)(P / \mathrm{s})^{-1 / 3}$ and $\mathrm{d} t / \mathrm{d} P=\left(\tau_{0}(n-\right.$ 1) $\left./ P_{0}\right)\left(1+t / \tau_{0}\right)^{1-1 /(n-1)}$.

The present proton energy spectrum has contributions both of neutron and proton components, the former is result of accumulation since the pulsar birth and the latter is the contribution at given time (or $P$ ). Therefore, the total proton spectrum can be written as

$\frac{\mathrm{d} N_{\mathrm{p}}}{\mathrm{d} E}=\left[\frac{\mathrm{d} N_{\mathrm{p}}\left(\gamma_{\mathrm{p}}, t_{\mathrm{CN}}\right)}{\mathrm{d} E}\right]_{1}+\left[\frac{\mathrm{d} N_{\mathrm{p}}\left(\gamma_{\mathrm{p}}, t_{\mathrm{CN}}\right)}{\mathrm{d} E}\right]_{2}$.

In Fig. 2, the total proton energy spectrum (solid line) is shown, which is the sum of the proton spectrum (dashed line) from neutron decay and the direct proton spectrum (dotted line). Although the cross section of neutrons and protons is similar, the present proton spectrum at low energy is mainly the contribution of low energy neutron decay accumulated in the pulsar evolution period, but at higher energy the direct proton emission contribution is greater than that of neutron decay. The results show that neglecting direct proton emission is not accurate. Following Bednarek \& Protheroe (1997), we can check whether the protons remain in the nebula or diffuse into the galactic environment; the typical diffusion distance to the present time is $x_{\mathrm{dif}} \approx$ $\left(c r_{\mathrm{L}} t_{\mathrm{CN}} / 3\right)^{1 / 2}$, where $r_{\mathrm{L}}$ is the Larmor radius of the protons, which is $r_{\mathrm{L}} \approx 10^{-3}\left(m_{\mathrm{p}} c^{2} / 940 \mathrm{MeV}\right)\left(\gamma_{\mathrm{p}} / 10^{5}\right)\left(B / 10^{-5} \mathrm{G}\right)^{-1} \mathrm{pc}$. For the protons with $\gamma_{\mathrm{p}}=10^{5}$ in a magnetic field of $B \sim 10^{-5} \mathrm{G}$, $x_{\text {dif }} \approx 0.36 \mathrm{pc}$, comparable to the radius of the nebula. Indeed the magnetic fields of the inner part of the Crab nebula near $0.2 \mathrm{pc}$ is even greater than $2 \times 10^{-3} \mathrm{G}$ measured in the synchrotron wisps, so protons with Lorentz factors $\gamma<5 \times 10^{5}$ could be trapped and accumulated in the Crab nebula.

\subsection{Leptonic spectra excited by nuclei}

In a PWN, leptons are excited by relativistic nuclei and consist of four components: positrons accelerated in a realistic magnetosonic lepton-heavy iron plasma shock, electrons from neutron decay, direct electrons, and secondary electron/positron pairs in a proton-proton interaction (Bednarek \& Bartosik 2003). We now give the spectra of the four kinds of leptons and show that 
the last three components are negligible compared to the first component.

For the first component, according to Hoshino et al. (1992), preferential non-thermal heating of positrons occurs in the realistic, magnetosonic, electron-positron-heavy ion shock. The gyrating reflected heavy ions dissipate their energy in the form of left-handed magnetosonic waves which are resonantly absorbed by the positron behind the iron reflection region. The positron energy spectrum can be described by a Maxwellian distribution with a supra-thermal power-law tail, i.e. (Hoshino et al. 1992)

$\left[\frac{\mathrm{d} N}{\mathrm{~d} E_{0} \mathrm{~d} t}\right]_{1}=\frac{N^{*}}{m_{\mathrm{e}} c^{2}}\left\{\frac{\gamma}{\gamma_{1}} \exp \left[-\frac{m_{\mathrm{e}} c^{2}}{T_{\mathrm{e}}}(\gamma-1)\right]+F(\gamma)\right\}$

with

$F(\gamma)=n_{\text {tail }}\left(\frac{\gamma}{\gamma_{1}}\right)^{-\alpha} G(\gamma)$ for $\gamma_{\min } \leq \gamma \leq \gamma_{\max }$,

and $G(\gamma)=1-\exp \left[-\left(\frac{\gamma-1}{\gamma_{\min }}\right)^{r}\right]$, for $r \gg 1$. The cutoff function becomes a step function at $\gamma=\gamma_{\mathrm{min}}$, where $\gamma_{\mathrm{min}}=\gamma_{\mathrm{Fe}}$ and $\gamma_{\max } \approx \gamma_{\mathrm{Fe}}(A / Z)\left(m_{\mathrm{i}} / m_{\mathrm{e}}\right), m_{\mathrm{e}}$ and $m_{\mathrm{i}}$ are the electron and nucleon mass (Bednarek 2003). $n_{\text {tail }}=0.076$ for $\alpha=2.4$ (Hoshino et al. 1992). To fit the observed radio emission we adopt $\gamma_{1} \approx 0.14 \gamma_{\mathrm{Fe}}$, and $T_{\mathrm{e}} / \gamma_{1} m_{\mathrm{e}} c^{2} \approx 0.14 . N^{*}$ is the normalization coefficient and can be estimated by using

$\int\left[\mathrm{d} N / \mathrm{d} E_{0} \mathrm{~d} t\right]_{1} E_{0} \mathrm{~d} E_{0}=\chi \dot{N}_{\mathrm{Fe}} E_{\mathrm{Fe}}$

where $\chi$ is the fractional energy of Fe transferred to positrons.

For the second component, electrons are produced through the neutron decay disintegrated from nuclei $\left(n \rightarrow p+\mathrm{e}^{-}+\bar{v}_{\mathrm{e}}\right)$, where their Lorentz factors are assumed to be equal to those of their parent nuclei. In this case, the electron spectrum can be approximated as

$\left[\frac{\mathrm{d} N}{\mathrm{~d} E_{0} \mathrm{~d} t}\right]_{2} \approx \frac{m_{\mathrm{n}}}{m_{\mathrm{e}}} \dot{N}_{\mathrm{Fe}} N_{\mathrm{n}}\left(E_{\mathrm{n}}\right)\left[1-\exp \left(v t / c \gamma_{\mathrm{n}}(t) \tau_{\mathrm{n}}\right)\right]$,

where $E_{0}=\gamma_{\mathrm{n}} m_{\mathrm{e}} c^{2}$ is the energy of injected electrons at time $t$, $N_{\mathrm{n}}\left(E_{\mathrm{n}}\right)$ is the emission neutron energy spectrum with Lorentz factor $\gamma_{\mathrm{n}}$, and the coefficient is because of the electron energy spectrum transformed from the decayed neutron spectrum (i.e. the same number electrons with the same Lorentz factor of a decayed neutron $\frac{\mathrm{d} E_{\mathrm{n}}}{\mathrm{d} E_{\mathrm{e}}}=\frac{m_{\mathrm{n}}}{m_{\mathrm{e}}}$.

According to Bednarek (2003), we consider the direct electron spectrum. We assume that the spin down energy transfer $1-\xi$ to monoenergy electrons with primary energy $E_{0}=$ $\gamma_{\mathrm{Fe}} m_{\mathrm{e}} c^{2}$, therefore the direct electron spectrum is given by

$\left[\frac{\mathrm{d} N}{\mathrm{~d} E_{0} \mathrm{~d} t}\right]_{3} \approx \frac{L(1-\xi)}{Z_{\mathrm{e}} \Phi(B, P)} \delta\left(E-E_{0}\right)$

where $Z_{\mathrm{e}}$ is the charge number of the electron. We also consider the spectrum of the secondary electron/positron pairs produced in proton-proton interaction $\left(p p \rightarrow \pi^{ \pm} \rightarrow \mu^{ \pm} \rightarrow \mathrm{e}^{ \pm}\right)$, but its contribution to the leptonic spectrum is negligible (Fig. 3).

Therefore, when we consider the leptons which consists of the first, second and third components, the leptonic spectrum is

$\frac{\mathrm{d} N}{\mathrm{~d} E_{0} \mathrm{~d} t}=\left[\frac{\mathrm{d} N}{\mathrm{~d} E_{0} \mathrm{~d} t}\right]_{1}+\left[\frac{\mathrm{d} N}{\mathrm{~d} E_{0} \mathrm{~d} t}\right]_{2}+\left[\frac{\mathrm{d} N}{\mathrm{~d} E_{0} \mathrm{~d} t}\right]_{3}$.

The leptons with an energy $E_{0}$ at an earlier time $t$ are injected into the nebula, and the energy $E$ of the leptons inside the nebula at the present time $t_{\mathrm{obs}}$ is determined by the energy loss rate and expansion of the nebula. Leptons injected into the nebula suffer mainly the following energy losses: bremsstrahlung, synchrotron, and inverse Compton losses as well as adiabatic loss

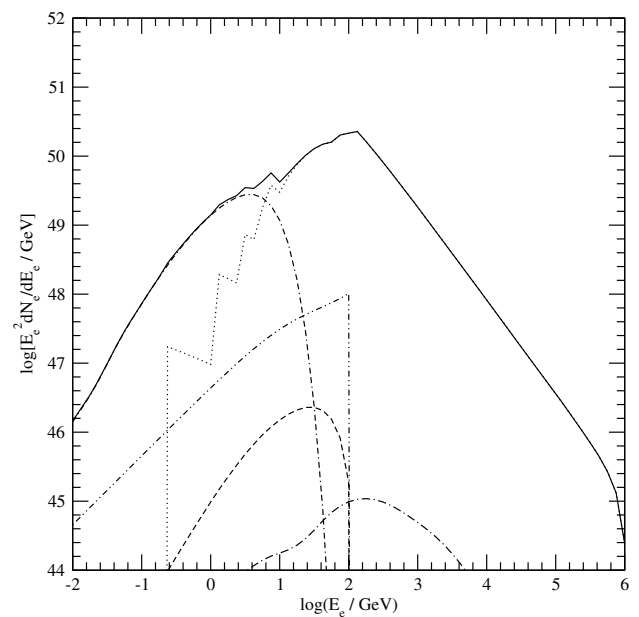

Fig. 3. Present spectra of leptons (solid line) in the Crab nebula. For comparison, we plot the spectra of the Maxwellian distribution (dashdash dotted line) and the power-law tail (dotted line); dashed, dot-dot dashed, and dot dashed lines represent the second to fourth component spectra.

due to the expansion of the nebula. The rate of their energy losses reads as

$-\frac{\mathrm{d} E}{\mathrm{~d} t}=\dot{E}_{\mathrm{brem}}+\dot{E}_{\mathrm{ad}}+\dot{E}_{\mathrm{syn}}+\dot{E}_{\mathrm{comp}}$,

where $\dot{E}_{\text {brem }} \approx m_{\mathrm{p}} N /\left(m_{\mathrm{e}} X\right) E$ describes the bremsstrahlung loss, where $X$ is the radiation length and $N$ is the density of the medium (Bednarek \& Bartosik 2003); $\dot{E}_{\text {ad }}=E \dot{R}_{\text {neb }} / R_{\text {neb }}$ is the adiabatic loss; $\dot{E}_{\mathrm{syn}}=(4 / 3) c \sigma_{\mathrm{T}} \gamma_{\mathrm{e}}^{2} \beta_{\mathrm{e}}^{2} B_{\text {neb }}^{2} / 8 \pi$ represents the synchrotron energy loss, where $\sigma_{\mathrm{T}}$ is the Thomson cross section and $B_{\text {neb }}$ is the magnetic field strength. For the inverse Compton scattering (ICS), leptons will lose energy by Compton upscattering ambient soft photon fields. Soft photons include the synchrotron radiation created by the leptons in the magnetic field of the nebula, the cosmic microwave background field, and the infrared photons produced by the the dust inside the nebula. The energy losses of leptons during the ICS in the "Klein-Nishina regime" can be safely neglected relative to the synchrotron energy losses at the energy range in our paper. For a realistic electron or positron traversing a blackbody photon gas with temperature $T$, the energy loss rate is described by Blumenthal \& Gould (1970) in the Thomson limit with a lowest-order correction with realistic quantum electrodynamics (Klein-Nishina formula); it is applicable in the Thomson and near Klein-Nishina regimes. We adopted the formula given by Gould (1975) $\dot{E}_{\mathrm{comp}}=4 / 3 \sigma_{\mathrm{T}} a c T^{2} \gamma_{\mathrm{e}}^{2}\left(1-24.15 \gamma_{\mathrm{e}} k T /\left(m_{\mathrm{e}} c^{2}\right)\right.$, where $a=7.5678 \times 10^{-15} \mathrm{erg} \mathrm{cm}^{-3} \mathrm{~K}^{-4}$; the last factor in the equation accounts for deviation from the Thomson limit when the deviation is small.

The leptonic spectrum at the present time is obtained by summing over the spectra from leptons injected at an early time and over all the time up to the time of the present observation, which is given by Bednarek \& Bartisik (2003) (also see Bednarek 2007):

$\frac{\mathrm{d} N\left(t_{\mathrm{obs}}\right)}{\mathrm{d} E}=\int_{0}^{t_{\mathrm{obs}}} J\left(t^{\prime}\right) \frac{\mathrm{d} N}{\mathrm{~d} E_{0} \mathrm{~d} t} \mathrm{~d} t$

where $\mathrm{d} N / \mathrm{d} E_{0} \mathrm{~d} t$ is the injection spectrum (Eq. (17)) of the leptons at time $t, t^{\prime}=t_{\mathrm{obs}}-t$ and $J\left(t^{\prime}\right)=E_{0} / E$. In Fig. 3, we show the current spectra of the leptons inside the nebula after the birth of the Crab pulsar, where the solid line represents the total spectrum. For comparison, we plot the spectra of the Maxwellian distribution (dash-dash dotted line) and the powerlaw tail (dotted line). In Fig. 3, dashed, dot-dot dashed, and dot 
dashed lines represent the second to fourth component spectra. It can be seen that the Maxwell component dominates in the lower energy range and the power law component dominates in higher energy range.

\section{Photon emission inside the nebula}

Having calculated the spectra of both leptons and hadrons, which depend on the time of evolution after the supernova explosion, we can compute the photon spectra produced by the leptons in different radiation processes and the hadrons in $p p$ interactions inside the nebula. The main leptonic processes which produce photons inside the nebula include synchrotron radiation, bremsstrahlung, and inverse Compton (IC) scattering. It is possible that photons produced by inverse Compton scattering of the high-energy leptons on synchrotron photons, microwave background photons, and infrared photons reach to very high energies. Despite different accuracies of IC cross section determination, as well as different distributions of magnetic fields and photon fields in nebula used in the past by different authors, the conclusion is unambiguous that the lepton component has a significant contribution to $\mathrm{TeV} \gamma$ rays (e.g. Gould 1965; Jones 1968; Rieke \& Weekes 1969; Kennel et al. 1984; de Jager \& Harding 1992; Atoyan \& Aharonian 1996; Zhang et al. 2008). On the other hand, hadrons produce high-energy photons and neutrinos in $p-p$ interactions. Below, we briefly describe the relevant processes and give our results for the Crab nebula.

\subsection{Photons produced by leptonic processes}

For the synchrotron radiation, the emissivity is given by

$$
\begin{aligned}
Q_{\text {syn }}\left(E_{\gamma}, t\right)= & \frac{2 \sqrt{3} \mathrm{e}^{3} B}{\hbar E_{\gamma} m_{\mathrm{e}} c^{3}} \int_{0}^{\pi / 2} \mathrm{~d} \theta \sin ^{2} \theta \\
& \times \int_{E_{\mathrm{e}, \min }}^{E_{\mathrm{e}, \max }} \mathrm{d} E_{\mathrm{e}} J_{\mathrm{e}}\left(E_{\mathrm{e}}, t\right) F\left(E_{\gamma} / E_{\mathrm{c}}\right)
\end{aligned}
$$

where $J_{\mathrm{e}}\left(E_{\mathrm{e}}\right)=(c \beta / 4 \pi) \mathrm{d} n\left(t_{\mathrm{obs}}\right) / \mathrm{d} E$, where $\mathrm{d} n\left(t_{\mathrm{obs}}\right) / \mathrm{d} E$ is the differential particle density over the nebula volume, $\theta$ is the electron pitch angle, $E_{\mathrm{c}}=(3 / 2) \gamma^{2}\left(h e B \sin \theta / m_{\mathrm{e}} c, B\right.$ is the local magnetic field strength, and $F(x)=x \int_{x}^{\infty} K_{5 / 3}(t) \mathrm{d} t$ with $x=E_{\gamma} / E_{\mathrm{c}}$, where $K_{5 / 3}(t)$ is the modified Bessel function of order 5/3 (Blumenthal 1970).

The electron-nucleon bremsstrahlung emissivity can be written as

$Q_{\text {brem }}\left(E_{\gamma}, t\right)=4 \pi n_{\mathrm{H}} \int_{E_{\mathrm{e}, \text { min }}}^{E_{\mathrm{e}, \text { max }}} \mathrm{d} E_{\mathrm{e}} J_{\mathrm{e}}\left(E_{\mathrm{e}}, t\right)\left(\frac{\mathrm{d} \sigma}{\mathrm{d} E_{\gamma}}\right)_{\mathrm{e}, \mathrm{p}}$,

where $\left(\mathrm{d} \sigma / \mathrm{d} E_{\gamma}\right)_{\mathrm{e}, \mathrm{p}}$ is the differential electron-proton bremsstrahlung cross section, which is (Koch \& Motz 1959)

$$
\begin{aligned}
\left(\frac{\mathrm{d} \sigma}{\mathrm{d} E_{\gamma}}\right)_{\mathrm{e}, \mathrm{p}}\left(E_{\mathrm{e}}, E_{\gamma}\right)= & \frac{r_{0}^{2} \gamma_{f} \beta_{f}}{137 E_{\gamma} \gamma_{i} \beta_{i}}\left(\frac{4}{3}-2 \gamma_{i} \gamma_{f}\left(\frac{\gamma_{i}^{2} \beta_{i}^{2}+\gamma_{f}^{2} \beta_{f}^{2}}{\gamma_{i}^{2} \beta_{i}^{2} \gamma_{f}^{2} \beta_{f}^{2}}\right)\right. \\
& +\frac{\alpha_{i} \gamma_{f}}{\gamma_{i}^{3} \beta_{i}^{3}}+\frac{\alpha_{f} \gamma_{i}}{\gamma_{f}^{3} \beta_{f}^{3}}-\frac{\alpha_{i} \alpha_{f}}{\gamma_{i} \beta_{i} \gamma_{f} \beta_{f}} \\
& +L\left\{\frac{8}{3 \beta_{i} \beta_{f}}+\frac{\epsilon_{\gamma}^{2}\left(1+\beta_{i}^{2} \beta_{f}^{2}\right)}{\gamma_{i} \beta_{i}^{3} \gamma_{f} \beta_{f}^{3}}\right. \\
& +\frac{\epsilon_{\gamma}}{2 \gamma_{i} \beta_{i} \gamma_{f} \beta_{f}}\left[\frac{\alpha_{i}\left(\gamma_{f}+\gamma_{i} \beta_{i}^{2}\right)}{\gamma_{i}^{2} \beta_{i}^{3}}-\frac{\alpha_{f}\left(\gamma_{i}+\gamma_{f} \beta_{f}^{2}\right)}{\gamma_{f}^{2} \beta_{f}^{3}}\right. \\
& \left.\left.\left.+\frac{2 \epsilon_{\gamma}}{\gamma_{i} \beta_{i}^{2} \gamma_{f} \beta_{f}^{2}}\right]\right\}\right)
\end{aligned}
$$

with

$\alpha_{i(f)}=\ln \left[\gamma_{i(f)}^{2}\left(1+\beta_{i(f)}\right)^{2}\right]$

and

$L=2 \ln \left(\frac{\gamma_{i} \gamma_{f}+\gamma_{i} \beta_{i} \gamma_{f} \beta_{f}-1}{\epsilon_{\gamma}}\right)$,

where $\gamma_{i(f)}$ is the initial(final) electron Lorentz factor, the dimensionless photon energy $\epsilon_{\gamma}=E_{\gamma} / m_{\mathrm{e}} c^{2}$, and $r_{0}=\mathrm{e}^{2} / m_{\mathrm{e}} c^{2}$ is the classical electron radius.

For the inverse Compton process, we use the full KleinNishina cross section for relativistic electrons to calculate the emissivity. The emissivity can be written as

$$
\begin{aligned}
Q_{\text {comp }, \mathrm{j}}\left(E_{\gamma}, t\right)= & 4 \pi \int_{0}^{\infty} \mathrm{d} \epsilon n_{j}(\epsilon, r) \int_{E_{\mathrm{e}, \mathrm{thresh}}}^{E_{\mathrm{e}, \max }} \\
& \times \mathrm{d} E_{\mathrm{e}} J_{\mathrm{e}}\left(E_{\mathrm{e}}, t\right) F\left(\epsilon, E_{\gamma}, E_{\mathrm{e}}\right),
\end{aligned}
$$

where $E_{\mathrm{e}, \text { thresh }}=\left[E_{\gamma}+\left(E_{\gamma}^{2}+E_{\gamma}\left(m_{\mathrm{e}} c^{2}\right)^{2} / \epsilon\right)^{1 / 2}\right] / 2$ is the lowest energy that electrons can scatter a target photon with energy $\epsilon$ to energy $E_{\gamma}, \epsilon$ is the target photon energy, and $n_{\mathrm{j}}$ is the number density of the soft photon component $j$. The energy distributions of the components of cosmic microwave background (CMB), IR and star light can be given by

$n_{\mathrm{j}}(\epsilon)=\frac{15 U_{\mathrm{j}}}{\left(\pi k T_{\mathrm{j}}\right)^{4}} \frac{\epsilon^{2}}{\exp \left(\epsilon / k T_{\mathrm{j}}\right)-1}$.

The energy density of the synchrotron photons is (Atoyan \& Aharonian 1996)

$n_{\mathrm{syn}}(r, \epsilon)=\frac{Q_{\mathrm{syn}}(\epsilon)}{4 \pi r_{0}^{2} c} U(x)$,

with

$U(x)=\frac{3}{2} \int_{0}^{1} \frac{y}{x} \ln \frac{x+y}{|x-y|} \mathrm{d} y$,

where $x \equiv r / r_{0}, Q_{\text {syn }}(\epsilon)$ is the synchrotron emissivity. In Eq. (27), it is assumed that the synchrotron emission is homogenous in the nebula with an average emission volume $V_{0}=\frac{4}{3} \pi r_{0}^{3}$. Function $F\left(\epsilon, E_{\gamma}, E_{\mathrm{e}}\right)$ is given by

$$
\begin{aligned}
F\left(\epsilon, E_{\gamma}, E_{\mathrm{e}}\right)= & \frac{3 \sigma_{\mathrm{T}}}{4\left(E_{\mathrm{e}} / m_{\mathrm{e}} c^{2}\right)^{2}} \frac{1}{\epsilon}[2 q \ln q+(1+2 q)(1-q) \\
& \left.+\frac{(\Gamma q)^{2}(1-q)}{2(1+\Gamma q)}\right]
\end{aligned}
$$

with $\Gamma=4 \epsilon\left(E_{\mathrm{e}} / m_{\mathrm{e}} c^{2}\right) / m_{\mathrm{e}} c^{2}, q=E_{1} / \Gamma\left(1-E_{1}\right)$ with $E_{1}=E_{\gamma} / E_{\mathrm{e}}$ and $1 / 4\left(E_{\mathrm{e}} / m_{\mathrm{e}} c^{2}\right)<q<1$.

\subsection{Photons and neutrinos produced by hadronic processes}

The protons accumulated in a PWN can generate high-energy photons and neutrinos in $p-p$ interactions $\left(p p \rightarrow p p+n_{\pi} \pi^{ \pm, 0}\right.$, where $n_{\pi}$ is the pion multiplicity). In this interaction process, high-energy photons are produced through the decay of neutral pions. Kelner et al. (2006) presented a simple analytical approximation with very good accuracy for the energy distribution of secondary products in the energy rangy above $0.1 \mathrm{TeV}$ and proposed an approximate procedure for the calculations toward 
Table 1. Main parameters for calculating photon and neutrino production.

\begin{tabular}{cccccccccc}
\hline \hline$\xi$ & $\chi$ & $\alpha$ & $B_{\text {neb }}(\mathrm{G})$ & $I\left(\mathrm{~g} \mathrm{~cm}^{2}\right)$ & $\tau_{0}(\mathrm{yr})$ & $\operatorname{Age}(\mathrm{yr})$ & $n_{\mathrm{H}}\left(\mathrm{cm}^{-3}\right)$ & $r_{0}(\mathrm{pc})$ & $d(\mathrm{pc})$ \\
\hline 0.85 & 0.42 & 2.4 & $4 \times 10^{-4}$ & $10^{45}$ & 500 & 1000 & 38 & 1.0 & 2000 \\
\hline
\end{tabular}

lower energies. Here we use energy-dependent parameterizations of the inelastic total cross section and differential $\pi^{0}$ production for proton-proton interactions (Kelner et al. 2006) to calculate the differential photon flux. For the higher energy region of $E_{\mathrm{p}}>0.1 \mathrm{TeV}$ and $E_{\gamma} / E_{\mathrm{p}} \geq 10^{-3}$, the photon emissivity can be expressed as

$$
\begin{aligned}
Q_{\pi}^{\mathrm{pp}}\left(E_{\gamma}\right)= & 4 \pi c n_{\mathrm{H}} \int_{E_{\gamma}}^{\infty} \sigma_{\text {inel }}\left(E_{\mathrm{p}}\right) J_{\mathrm{p}}\left(E_{\mathrm{p}}, t\right) \\
& \times F_{\gamma}\left(\frac{E_{\gamma}}{E_{\mathrm{p}}}, E_{\mathrm{p}}\right) \frac{\mathrm{d} E_{\mathrm{p}}}{E_{\mathrm{p}}}
\end{aligned}
$$

where $J_{\mathrm{p}}\left(E_{\mathrm{p}}, t\right)=(c \beta / 4 \pi) \mathrm{d} N_{\mathrm{p}}\left(E_{\mathrm{p}}\right) / \mathrm{d} E_{\mathrm{p}}, n_{\mathrm{H}}$ is the density of the ambient hydrogen, $\sigma_{\text {inel }}\left(E_{\mathrm{p}}\right)$ is the inelastic part of the total cross section of $p-p$ interactions, which is described as

$\sigma_{\text {inel }}\left(E_{\mathrm{p}}\right)=34.3+1.88 L+0.25 L^{2} \times\left[1-\left(\frac{E_{\mathrm{th}}}{E_{\mathrm{p}}}\right)^{4}\right]^{2} \mathrm{mb}$

where $E_{\mathrm{th}}$ is the threshold energy of production of neutral pions, where $L=\ln \left(E_{\mathrm{p}} / 1 \mathrm{TeV}\right) . F_{\gamma}\left(x, E_{\mathrm{p}}\right)$ is the number of photons in the interval $(x, x+\mathrm{d} x)$ per collision, which is given in Eq. (58) of Kelner et al. (2006), where $x=E_{\gamma} / E_{\mathrm{p}}$. For the lower energy region of $1 \mathrm{GeV} \ll E_{\mathrm{p}} \leq 100 \mathrm{GeV}$, Aharonian \& Atoyan (2000) proposed that the photon spectrum can be treated using a $\delta$-functional approximation and the photon emissivity in this energy region can be written as

$Q_{\pi}^{\mathrm{pp}}\left(E_{\gamma}\right)=2 \int_{E_{\pi, \text { min }}}^{\infty} \mathrm{d} E_{\pi} \frac{q_{\pi}\left(E_{\pi}, t\right)}{\sqrt{E_{\pi}^{2}-m_{\pi} c^{2}}}$,

where $E_{\pi, \min }=E_{\gamma}+m_{\pi}^{2} c^{4} / 4 E_{\gamma}$, and

$$
\begin{aligned}
q_{\pi}\left(E_{\pi}, t\right)= & \tilde{n} \frac{4 \pi n_{\mathrm{H}}}{K_{\pi}} \sigma_{\text {inel }}\left(m_{\mathrm{p}} c^{2}+E_{\pi} / K_{\pi}\right) \\
& \times J_{\mathrm{p}}\left(m_{\mathrm{p}} c^{2}+E_{\pi} / K_{\pi}, t\right),
\end{aligned}
$$

where $\tilde{n}$ and $K_{\pi}$ are parameters that depend weakly on the proton energy. Kelner et al. (2006) pointed out that the photon emissivity in the energy range $E_{\mathrm{p}} \geq 0.1 \mathrm{TeV}$ is calculated using Eq. (30), and at lower energy using Eq. (33) with $K_{\pi}=0.17$. The parameter $\tilde{n}$ is treated as a free parameter determined from the conditions that match the emissivity given by Eq. (30) at $E_{\mathrm{p}}=0.1 \mathrm{TeV}$.

In the $p-p$ collision, the pion emissivities satisfy the following relationship: $Q_{\pi^{0}} \approx Q_{\pi^{+}} \approx Q_{\pi^{-}}$. The muonic neutrinos are produced at the decay of charged pions $\pi \rightarrow \mu v_{\mu}$, and the corresponding third particle decay yields muonic and electronic neutrinos as the mode $\mu \rightarrow e v_{\mathrm{e}} \nu_{u}$. Taking neutrino oscillations into account, all muonic neutrinos and electronic neutrinos are incorporated and the emissivity of the muonic neutrinos is

$Q_{v_{\mu}}=P_{\mu \mu} Q_{v_{\mu}}+P_{\mathrm{e} \mu} Q_{v_{\mathrm{e}}}$,

where $P_{\mu \mu}=0.39$ and $P_{\mu \mu}=0.22$ are the oscillation probability and the emissivity of muonic neutrinos and electronic neutrinos are calculated by using the formulae given by Kelner et al. (2006). In our calculations, we did not consider the neutrino from neutron decay $p \rightarrow p+\mathrm{e}^{-}+\bar{v}_{\mathrm{e}}$ since the laboratory energy is low(estimated by $\left.E_{v}=\gamma \Delta, \Delta=m_{\mathrm{n}}-m_{\mathrm{p}}-m_{\mathrm{e}}=0.71 \mathrm{MeV}\right)$, it mainly contributes to the energy below several ten $\mathrm{GeV}$.

\subsection{Calculation results}

We now calculate the photon spectrum and muonic neutrino flux for the Crab nebula. For the photons produced in the nebula, the spectrum observed on the Earth can be expressed as

$$
\begin{aligned}
\frac{\mathrm{d} N}{\mathrm{~d} E_{\gamma}}= & \frac{V_{\text {neb }}}{4 \pi d^{2}}\left[Q_{\text {syn }}\left(E_{\gamma}\right)+Q_{\text {brem }}\left(E_{\gamma}\right)\right. \\
& +Q_{\text {comp }, \mathrm{j}}\left(E_{\gamma}\right)+Q_{\pi}^{\mathrm{pp}}\left(E_{\gamma}\right],
\end{aligned}
$$

where $V_{\text {neb }}$ is the volume of the nebula and $d$ is the distance from the earth to the nebula. The spectrum of synchrotron and Compton radiation integrated over the nebula volume is independent of $R_{\mathrm{neb}}$, but the bremsstrahlung and $p p$ production is effected by $n_{\mathrm{H}}=M / V_{\text {neb }} m_{\mathrm{p}}$; radio and optical observations indicate that the nebula has an angular size of $5 \times 7 \mathrm{arcmin}^{2}$, which corresponds to a spatial size of $3 \times 4 \mathrm{pc}^{2}$ for $d=2 \mathrm{kpc}$. Protons are efficiently trapped by the dense filaments as suggested by Atoyan \& Aharonian (1996) and filaments with a density $\sim 500 \mathrm{~cm}^{-3}$ exist in the Crab nebula (Davidson \& Fesen 1985). Therefore we use $n_{\mathrm{H}}=38 \mathrm{~cm}^{-3}$ which is slightly greater than the averaged density in our calculation. For the muonic neutrinos produced in the nebula, the the neutrino flux observed on the Earth is

$F_{v_{\mu}}(E)=\frac{V_{\text {neb }}}{4 \pi d^{2}} Q_{v_{\mu}}$.

We calculated the multiwaveband photon and high energy neutrino spectra of the Crab nebula and the main parameters used here are listed in Table 1. In Fig. 4, we show the comparison of the predicted photon spectrum with the observed data for the Crab nebula. The radio data are taken from Baars \& Hartsuijker (1972), millimeter and IR data from Strom \& Greidanus (1992), Bandiera et al. (2002), and Green et al. (2004), soft X-ray to $\gamma$ ray data from Kuiper et al. (2001), and VHE $\gamma$-ray data from Aharonian et al. (2004, 2006) and Albert et al. (2008). In Fig. 5, we address the comparison of the predicted result with observed high-energy data. It can be seen that our results can reproduce the observed multi-waveband spectrum well. Our results indicate that the photon spectrum up to $\sim 10 \mathrm{TeV}$ has a leptonic origin, but the spectrum with energy greater than $10 \mathrm{TeV}$ consists of both hadronic and leptonic components.

In Fig. 6, we show the predicted muonic neutrino flux observed on the Earth and the atmospheric neutrino background (Lipari 1993) within $1^{\circ}$ as indicated by the shaded band. Compared to the result given by Bednarek (2003), our result is slightly lower. Bednarek (2003) estimated that the number of $\mu$ neutrinos observed by the $1 \mathrm{~km}^{2}$ detector during 1 year is about 1.3 for the Crab nebula.

\section{Discussion and conclusion}

We have studied multi-waveband photon emission and possible high-energy neutrino emission from the Crab nebula in the framework of the hadronic-leptonic model. In this model, we considered nucleon injection in the pulsar magnetosphere, proton production by the photo-disintegration process, and leptonic production excited by the nuclei in detail. The proton spectrum 


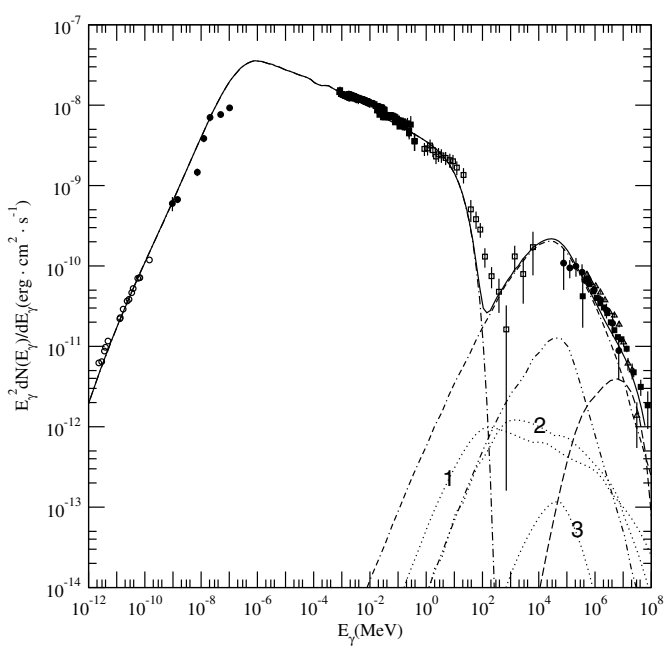

Fig. 4. Comparison of the calculated spectrum with observed data for the Crab nebula. The radio data are taken from Baars \& Hartsuijker (1972), millimeter and IR data from Strom \& Greidanus (1992), Bandiera et al. (2002), and Green et al. (2004), soft X-ray to $\gamma$-ray data from Kuiper et al. (2001), and VHE $\gamma$-ray data from Aharonian et al. (2004, 2006) and Albert et al. (2008). Dash-dotted, dot-dot dashed, and dash-dash dotted curves represent the synchrotron, bremsstrahlung, and self synchrotron Compton emission spectra of the leptons, curves 1, 2, and 3 are the spectra of the inverse Compton scattering of the leptons with the microwave background, infrared photons, and optical photons, the dashed curve represents the $\pi^{0}$-decay spectrum in the $p p$ interaction, and the solid line is total emission.

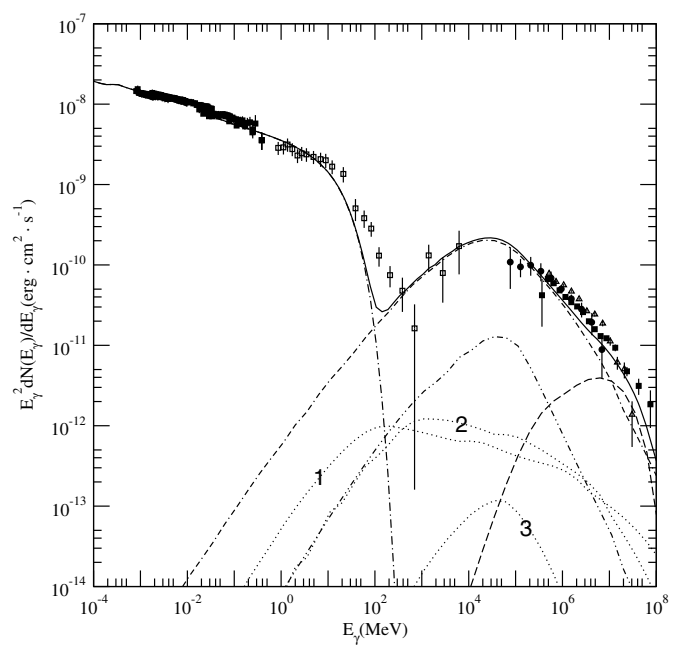

Fig. 5. Comparison of the calculated spectrum with observed highenergy data for the Crab nebula. Symbols are the same as in Fig. 4.

consists of two components: the proton component from neutron decay and the direct emitting proton component. The first component dominates over the second component in the lower energy region, but the second component has its main contribution in the higher energy region (see Fig. 2). It should be noted that previous works (e.g. Bednarek \& Prothero 1997; Bednarek $\&$ Bartosik 2003) did not consider the contribution of the second component. For the leptons excited by the nuclei, the spectrum consists of four components: positrons accelerated in realistic magneto-sonic lepton-heavy iron plasma shocks, electrons from neutron decay, direct electrons, and secondary electron/positron pairs in the $p p$ interaction (see Fig. 3), it can be seen from this figure that the first component dominates over other components, which is different from that given by

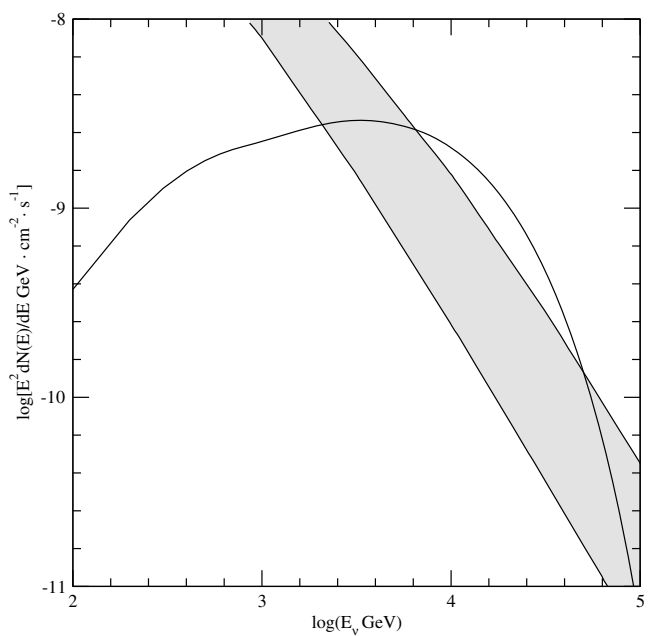

Fig. 6. Predicted spectrum of muonic neutrinos observed on the Earth. The atmospheric neutrino background within $1^{\circ}$ of the source is indicated by the shaded band.

Bednarek \& Bartosik (2003) since the complete form of the positron spectrum given by Hoshino et al. (1992) is included in our calculation.

After determining the spectra of both hadrons and leptons, we calculated the multi-waveband photon spectrum of the Crab nebula (see Figs. 4 and 5). The calculated spectrum up to TeV energy could be explained by upscattering soft photon fields by realistic leptons accompanying nuclei, where the soft photon fields mainly include synchrotron radiation, microwave background, infrared and optical photons. However our predicted spectrum reveals that the hadronic component has an important contribution to gamma rays in the energy range above several TeV. Bednarek \& Bartosik (2003) found that the emission from the Crab nebula can be well fitted by the composition of the $\gamma$-ray emission produced by leptons $(<10 \mathrm{TeV})$ and nuclei $(>10 \mathrm{TeV})$. Compared to the results of Bednarek \& Bartosik (2003), our results show that it is difficult to clearly distinguish leptonic and hadronic origins in the $\sim 10 \mathrm{TeV}$ energy range (see Fig. 5). For the $\mu$ neutrino emission, our predicted flux (see Fig. 6) is lower than that given by Bednarek (2003).

We now discuss the possible influence of our treatments on the predicted photon and neutrino fluxes. In this paper, we have given the treatment of disintegration as accelerating along the magnetic line with a non-perpendicular electric field in the outer gap. The nucleon energy spectrum from nucleus disintegration is calculated simply by Eq. (6) assuming a constant electronic field. Because the real $\frac{\mathrm{d} s}{\mathrm{~d} \gamma_{A}}$ near the outer range of the gap should be larger than our average value, the calculation will mainly underestimate the the proton energy spectrum at high energy. If detailed information about $E_{\|}(s)=f(s)$ is known, the nucleon energy spectrum could be calculated precisely as could its more distinct contribution to the very high photon spectrum. It should be noted that the direct proton contribution at high energy is significant irrespective of the calculation technique. Another factor which could effect the nucleon energy spectrum and thus the maximum positron energy is that the kinetic energy of nuclei entering the outer gap is assumed to be zero; this could lead to a decrease in the disintegration rate and thus nuclei maximum energy (or maximum positron energy), resulting in the decrease of fluxes at high energy of $p p$ photon emission and synchrotron radiation. 
Acknowledgements. This work is partially supported by the National Natural Science Foundation of China under grant 10778702 and a 973 Program (2009CB824800).

\section{References}

Aharonian, F. A., \& Atoyan, A. M. 1995, Astropart. Phys., 3, 275

Aharonian, F. A., \& Atoyan, A. M. 2000, A\&A, 362, 937

Aharonian, F., Akhperjanian, A., Beilicke, M., et al. 2004, ApJ, 614, 897

Aharonian, F., Akhperjanian, A. G., Bazer-Bachi, A. R., et al. 2006, A\&A, 457, 899

Albert, J., Aliu, E., Anderhub, H., et al. 2008, ApJ, 674, 1037

Alvarez, R. A., Berman, B. L., Faul, D. D., Lewis Jr, F. H., \& Meyer, P. 1979, Phys. Rev., Part C, Nucl. Phys., 20, 128

Atoyan, A. M., \& Aharonian, F. A., 1996, MNRAS, 278, 525

Baars, J. W., \& Hartsuijker, A. P. 1972, A\&A, 17, 172

Bandiera, R., Neri, R., \& Cesaroni, R. 2002, A\&A, 386, 1044

Bednarek, W. 2003, A\&A, 407, 1

Bednarek, W. 2007, Ap\&SS, 309, 179

Bednarek, W., \& Protheroe, R. J. 1997, Phys. Rev. Lett., 79, 2616

Bednarek, W., \& Bartosik, M. 2003, A\&A, 405, 689

Bednarek, W., \& Bartosik, M. 2004, A\&A, 423, 405

Bednarek, W., \& Bartosik, M. 2005, J. Phys. G Nucl. Phys., 31, 1465

Blumenthal, G. R., \& Gould, R. J. 1970, Rev. Mod. Phys., 42, 237

Cheng, K. S., Cheung, T., Lau, M. M., Yu, K. N. \& Kwok, P. W. 1990, J. Phys. G Nucl. Phys., 16, 1115

Cheng, K. S., Ho, C., \& Ruderman, M. 1986a, ApJ, 300, 500
Cheng, K. S., Ho, C., \& Ruderman, M. 1986b, ApJ, 300, 522 de Jager, O. C., \& Harding, A. K. 1992, ApJ, 396, 161

Danos, M., \& Fuller, E. G. 1965, Ann. Rev. Nucl. Part. Sci., 15, 29

Davidson, K., \& Fesen, R. A. 1985, ARA\&A, 23, 119

Gaensler, B. M., \& Slane, P. O. 2006, ARA\&A, 44, 17

Gallant, Y. A., \& Arons, J. 1994, ApJ, 435, 230

Gil, J., Melikidze, G. I., \& Geppert, U. 2003, A\&A, 407, 315

Gould, R. J. 1975, ApJ, 196, 689

Gould, R. J. 1965, Phys. Rev. Lett., 15, 577

Green, D. A., Tuffs, R. J., \& Popescu, C. C. 2004, MNRAS, 355, 1315

Hoshino, M., \& Arons, J. 1991, Phys. Fluids B, 3, 818

Hoshino, M., Arons, J., Gallant, Y. A., \& Langdon, A. B. 1992, ApJ, 390, 454

Jones, F. C. 1968, Phys. Rev., 167, 1159

Karakula, S., \& Tkaczyk, W. 1993, Astropart. Phys., 1, 229

Katz, L., \& Cameron, A. G. W. 1951, Canadian J. Phys., 29, 518

Kelner, S. R., Aharonian, F. A., \& Bugayov, V. V. 2006, Phys. Rev. D, 74, 034018

Kennel, C. F., \& Coroniti, F. V. 1984, ApJ, 283, 710

Kuiper, L., Hermsen, W., Cusumano, G., et al. 2001, A\&A, 378, 918

Koch, H. W., \& Motz, J. W. 1959, Rev. Mod. Phys., 31, 920

Lipari, P. 1993, Astropart. Phys., 1, 195

Lyne, A. G., Pritchard, R. S., \& Graham-Smith, F. 1993, MNRAS, 265, 1003

Puget, J. L., Stecker, F. W., \& Bredekamp, J. H. 1976, ApJ, 205, 638

Rieke, G. H., \& Weekes, T. C. 1968, ApJ, 155, 429

Ribordy, M., et al. (IceCube Collaboration) 2006, Physics of Atomic Nuclei, 69, 1899

Stecker, F. W., \& Salamon, M. H. 1999, ApJ, 512, 521

Strom, R. G., \& Greidanus, H. 1992, Nature, ,358, 654

Zhang, L., Chen, S. B., \& Fang, J. 2008, ApJ, 676, 1210 